\title{
Polarimeters and energy spectrometers for the ILC Beam Delivery System
}

\author{
S. Boogert, ${ }^{a}$ A.F. Hartin, ${ }^{b}$ M. Hildreth, ${ }^{c}$ D. Käfer, ${ }^{b}$ J. List, ${ }^{b, 1}$ T. Maruyama, ${ }^{d}$ K. Mönig, ${ }^{b}$ \\ K.C. Moffeit, ${ }^{d}$ G. Moortgat-Pick, ${ }^{e}$ S. Riemann, ${ }^{b}$ H.J. Schreiber, ${ }^{b}$ P. Schüler, ${ }^{b}$ \\ E. Torrence ${ }^{f}$ and M. Woods ${ }^{d}$ \\ ${ }^{a}$ The Physics Department, Royal Holloway University of London, \\ Egham, Surrey TW20 OEX, U.K. \\ ${ }^{b}$ DESY - Deutsches Elektronensynchrotron, \\ Notkestrasse 85, 22607 Hamburg and Platanenallee 6, 15738 Zeuthen, Germany \\ ${ }^{c}$ Department of Physics, University of Notre Dame, \\ 225 Nieuwland Science Hall, Notre Dame, IN 46556-5670, U.S.A. \\ ${ }^{d}$ SLAC National Accelerator Laboratory, \\ 2575 Sand Hill Road, Menlo Park, CA 94025, U.S.A. \\ ${ }^{e}$ Institute for Particle Physics Phenomenology, University of Durham, \\ South Rd, Durham DH1 3LE, U.K. \\ ${ }^{f}$ Physics Department, University of Oregon, \\ 1274 University of Oregon, Eugene, OR 97403-1274, U.S.A.
}

E-mail: jenny.list@desy.de

ABSTRACT: Any future high energy $e^{+} e^{-}$linear collider aims at precision measurements of Standard Model quantities as well as of new, not yet discovered phenomena. In order to pursue this physics programme, excellent detectors at the interaction region have to be complemented by beam diagnostics of unprecedented precision. This article gives an overview of current plans and issues for polarimeters and energy spectrometers at the International Linear Collider, which have been designed to fulfill the precision goals at a large range of beam energies from $45.6 \mathrm{GeV}$ at the $Z^{0}$ pole up to $250 \mathrm{GeV}$ or, as an upgrade, up to $500 \mathrm{GeV}$.

KEYWORDS: Instrumentation for particle accelerators and storage rings - high energy (linear accelerators, synchrotrons); Spectrometers; Accelerator modelling and simulations (multi-particle dynamics; single-particle dynamics)

ARXIV EPRINT: 0904.0122v2

\footnotetext{
${ }^{1}$ Corresponding author.
} 


\section{Contents}

1 Introduction and overview 1

2 Polarimetry 3

2.1 Polarimeter detectors $\quad 6$

$\begin{array}{lll}2.2 & \text { Upstream polarimeter } & 7\end{array}$

2.3 Downstream polarimeter 8

2.4 Impact of crossing angle and IR magnets on polarimetry 9

3 Beam energy measurements $\quad 10$

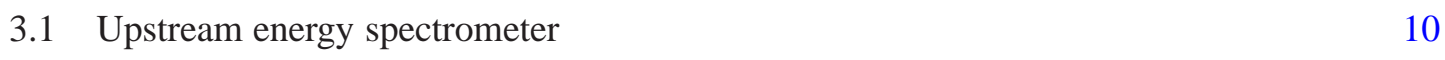

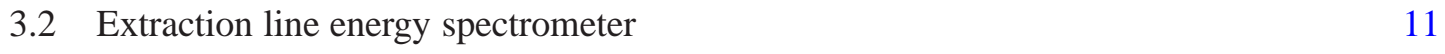

$\begin{array}{ll}3.3 & \text { Alternative methods for energy measurements } \\ \end{array}$

$\begin{array}{lll}4 & \text { Summary } & 13\end{array}$

\section{Introduction and overview}

The International Linear Collider (ILC) will open a new precision frontier, with beam polarization playing a key role in a physics program that demands precise polarization and beam energy measurements [1]. The baseline configuration of the ILC, as described in the Reference Design Report (RDR) [2], provides polarized electron and positron beams, with spin rotator systems to achieve longitudinal polarization at the collider interaction point (IP); upstream and downstream polarimeters and energy spectrometers for both beams; and the capability to rapidly flip the electron helicity at the injector, using the source laser. The possibility of fast positron helicity flipping is not included in the baseline configuration. A scheme for fast positron helicity flipping has been proposed [3].

The electrons will be highly polarized with $P\left(e^{-}\right)>80 \%$. Positrons will also be produced with an initial polarization $P\left(e^{+}\right) \sim 30-45 \%$, which can be upgraded to $60 \%$. Even the small positron polarization expected in the inital setup can be used with great benefit for physics measurements if the possibility of fast helicity flipping of the positron spin is also provided. Excellent polarimetry for both beams, accurate to $\Delta P / P=0.25 \%$, is planned [1,4]. Polarimetry will be complemented by $e^{+} e^{-}$collision data, where processes like $W^{ \pm}$pair production can provide an absolute scale calibration for the luminosity-weighted polarization at the IP, which can differ from the polarimeter measurements due to depolarization in collision.

Precise beam energy measurements are necessary at the ILC in order to measure particle masses produced in high-rate processes. Measuring the top mass in a threshold scan to order $100 \mathrm{MeV}$ or measuring a Standard Model Higgs mass in direct reconstruction to order $50 \mathrm{MeV}$ requires knowledge of the luminosity-weighted mean collision energy $\sqrt{s}$ to a level of $(1-2) \cdot 10^{-4}[1$, 4]. Precise measurements of the incoming beam energy are a critical component to measuring the quantity $\sqrt{s}$ as it sets the overall energy scale of the collision process. 
ILC e $e^{-}$BDS $(500 \mathrm{GeV})$
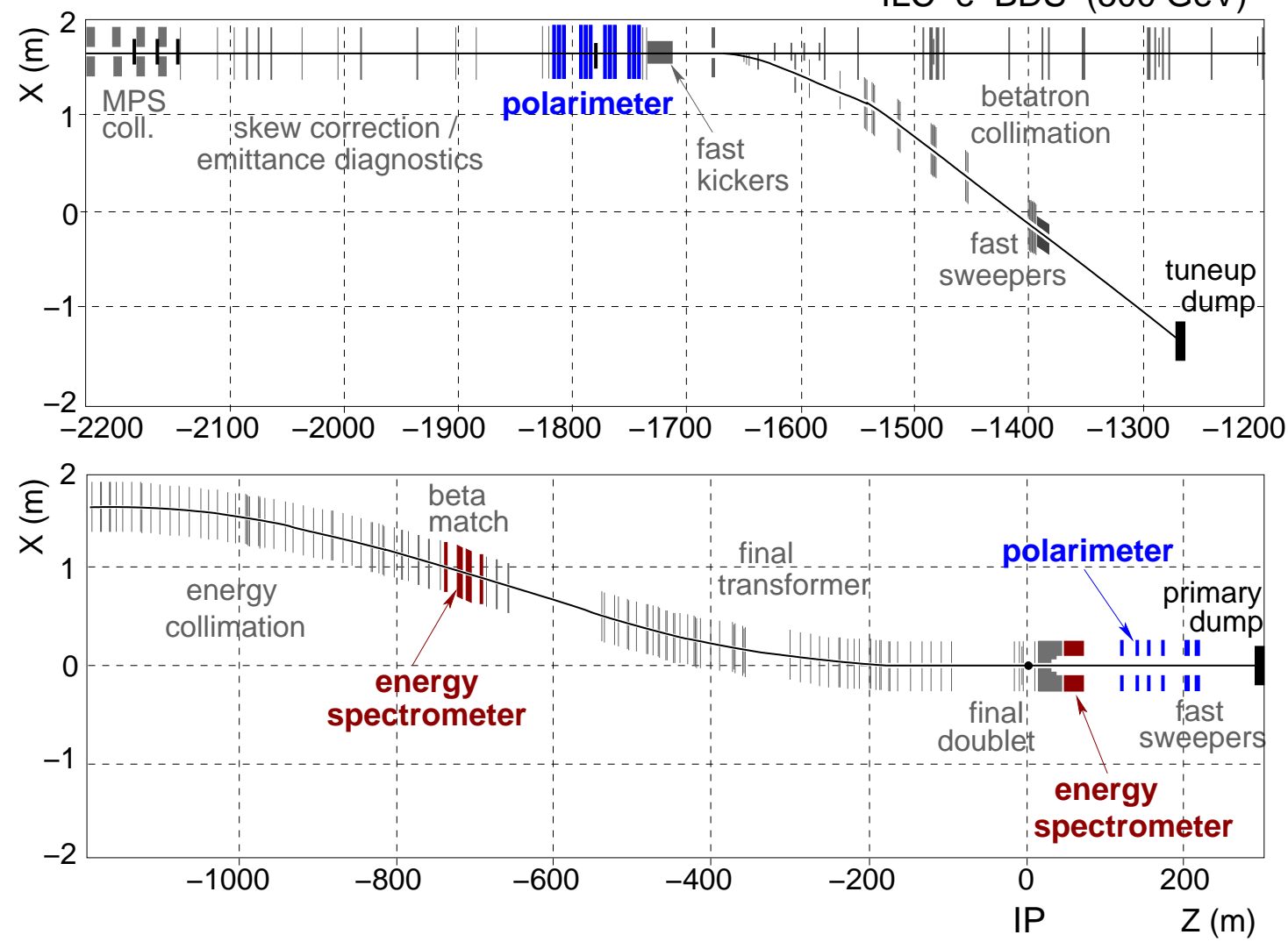

Figure 1. Beam Delivery System (BDS) as described in the RDR. The upper part shows the region from $2200 \mathrm{~m}$ to $1200 \mathrm{~m}$ upstream of the $e^{+} e^{-} \mathrm{IP}$, including the polarimeter chicane at $1800 \mathrm{~m}$. The lower part shows the region from $1200 \mathrm{~m}$ upstream to $400 \mathrm{~m}$ downstream of the IP, including the upstream energy spectrometer at $700 \mathrm{~m}$ as well as the extraction line energy spectrometer and polarimeter around $100 \mathrm{~m}$ downstream of the IP located at $z=0 \mathrm{~m}$.

The baseline ILC described in the RDR provides collider physics with beam energies in the range $100-250 \mathrm{GeV}$. Precise polarization and energy measurements are required for this full energy range. The ILC baseline also provides for detector calibration at the $Z$-pole with $45.6 \mathrm{GeV}$ beam energies. However, the RDR does not require accurate polarimetry or energy spectrometer measurements at the $Z$-pole. A proposal to modify the baseline ILC to require precise polarimetry and energy measurements at $Z$-pole energies was made at the Workshop on Polarization and Beam Energy Measurements at the ILC, held in Zeuthen in 2008 [4]. The motivation for this includes polarimeter and energy spectrometer calibration, and physics measurements to improve on $Z$-pole results from LEP and SLC. The downstream polarimeter described in the RDR is expected to perform well at the $Z$-pole, while for the upstream polarimeter the necessary changes are described in this paper and will be included in the next update of the ILC baseline design. For energy measurements, the downstream energy spectrometer should perform well while the upstream spectrometer needs further evaluation as to how accurately the lower chicane magnetic fields can be measured.

The locations of the polarimeters and energy spectrometers in the Beam Delivery System (BDS) as forseen in the RDR are shown in figure 1. Data from the polarimeters and spectrometers 
(a)

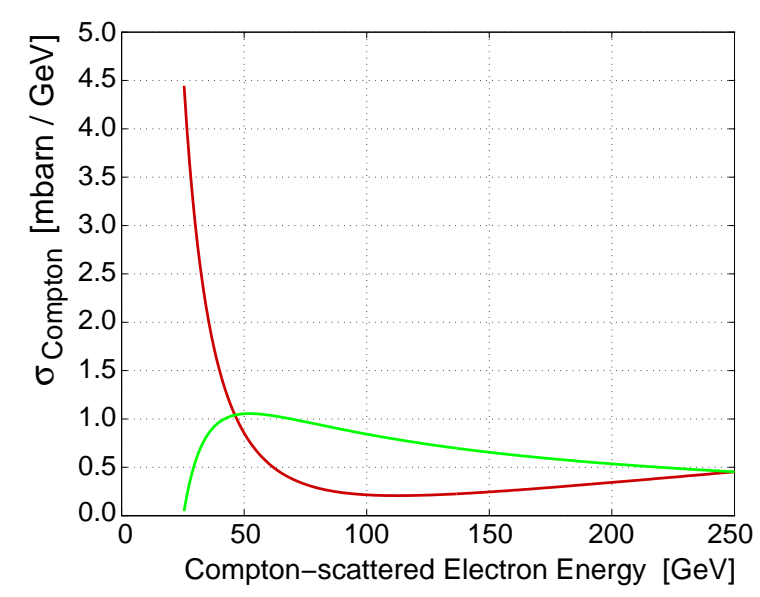

(b)

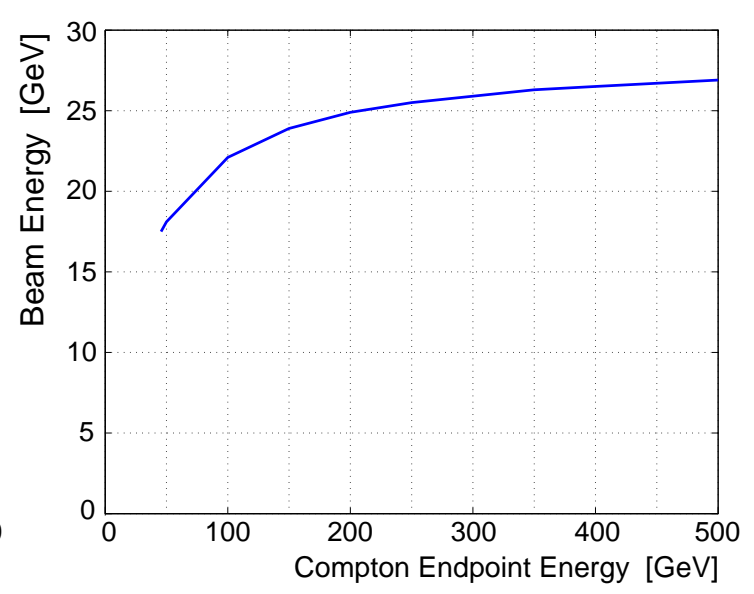

Figure 2. (a) Compton differential cross section versus scattered electron energy for same (black/red curve) and opposite (grey/green curve) helicity configuration of laser photon and beam electron. The beam energy is $250 \mathrm{GeV}$ and the laser photon energy is $2.3 \mathrm{eV}$. (b) Compton edge energy dependence on the beam energy.

must be delivered to the detector data acquisition system in real time to be logged and to permit fast online analysis. On the other hand, fast online analysis results must also be provided to the ILC control system for beam tuning and diagnostics.

The purpose of this paper is to describe the polarimeters and energy spectrometers which have been designed in order to fulfil the precision requirements over the entire range of beam energies from $45.6 \mathrm{GeV}$ up to $500 \mathrm{GeV}$. Section 2 provides an overview of the functional principle of the BDS polarimeters, discusses possible choices of Cherenkov detectors and the layouts of both magnetic chicanes, as well as the challenges caused by the forseen $14 \mathrm{mrad}$ crossing angle of electron and positron beams at the IP. Section 3 presents an overview of the layout and technology foreseen for the upstream and downstream energy spectrometers, while also providing a short subsection on alternative methods for beam energy measurements.

\section{Polarimetry}

Both upstream and downstream BDS polarimeters will use Compton scattering of high power lasers with the electron and positron beams [1,2]. Figure 2 shows the Compton cross section versus scattered electron energy for $250 \mathrm{GeV}$ beam energy and $2.3 \mathrm{eV}$ photon energy. There is a large polarization asymmetry for back-scattered electrons near $25.2 \mathrm{GeV}$, the Compton edge energy. The large asymmetry and the large difference between the Compton edge and the beam energy facilitate precise polarimeter measurements. The Compton edge does not change significantly for higher beam energies; this dependence is also shown in figure 2.

A spectrometer with segmented Cherenkov detectors that sample the flux of scattered electrons near the Compton edge will be used to provide good polarization measurements with high analyzing power. Compton polarimetry, utilizing measurements of back-scattered electrons near the Compton edge, is chosen as the primary polarimetry technique for several reasons: 


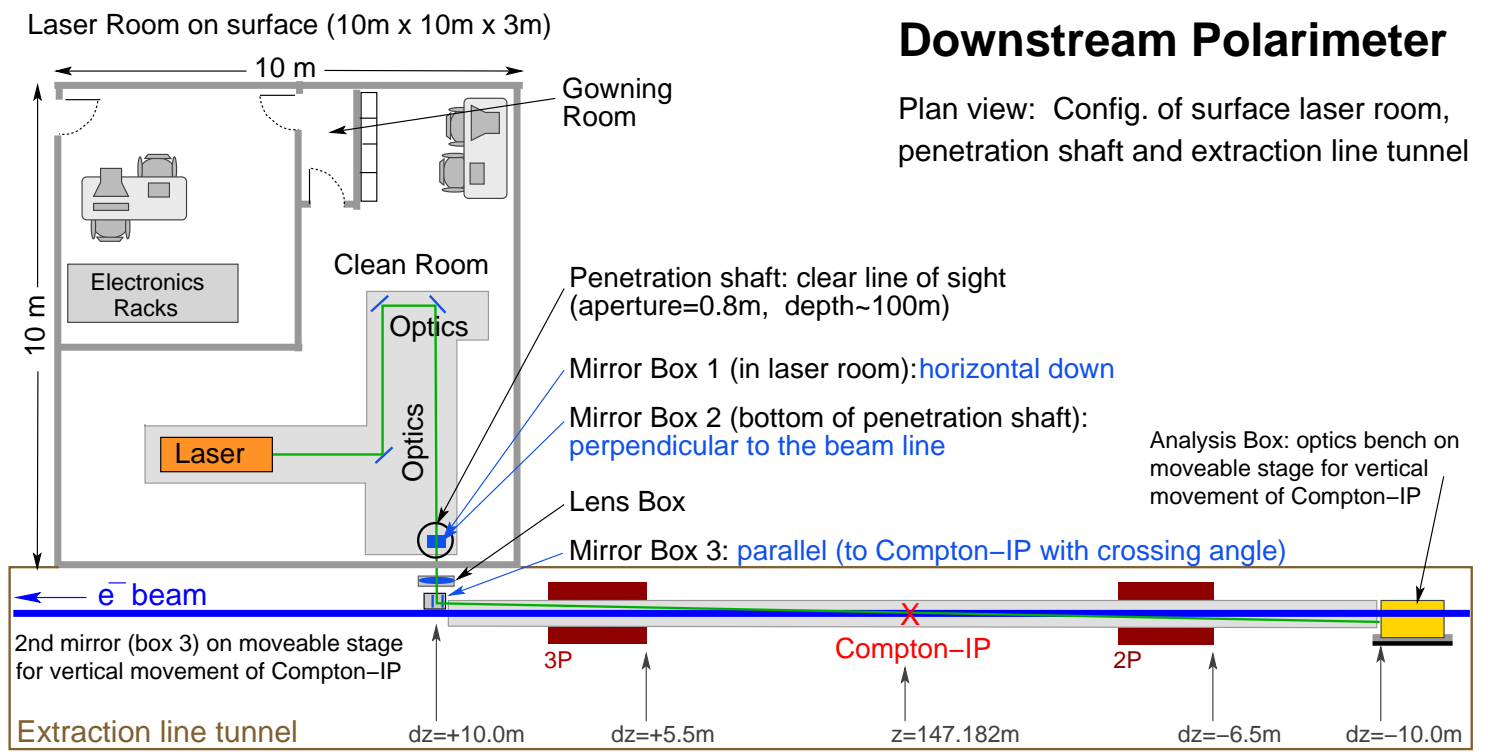

Figure 3. Proposed configuration of laser room, penetration shaft and extraction line layout for the downstream Compton polarimeter.

- The physics of the scattering process is well understood from QED, with radiative corrections of less than $0.1 \%$ [5];

- Detector backgrounds are easy to measure and correct for using "laser off" pulses;

- Compton-scattered electrons can be identified, measured and isolated from backgrounds using a magnetic spectrometer;

- Polarimetry data can be taken parasitic to physics data;

- The Compton scattering rate is high and small statistical errors can be achieved in a short amount of time (sub-1\% precision in one minute is feasible);

- The laser helicity can be selected on a pulse-by-pulse basis; and

- The laser polarization is readily determined with $0.1 \%$ accuracy.

It is expected that a systematic precision of $\Delta P / P=0.25 \%$ or better can be achieved with the largest uncertainties coming from the analyzing power calibration and the detector linearity, both of which are in the range of $0.1 \%$ to $0.2 \%$ [9].

Each polarimeter requires a laser room on the surface with a transport line to the beamline underground. A configuration proposed for the extraction line polarimeter is shown in figure 3. A similar configuration is planned for the upstream polarimeter. The entire layout of the laser room and penetration shaft is conceptual and still needs to be optimised to keep the radiation in the laser room below required levels even for a worse case scenario of beam loss. It is possible, for example, to pack the $80 \mathrm{~cm}$ wide penetration shaft with neutron absorbing material at the top and bottom, which (togehter with its depth of about $100 \mathrm{~m}$ ) would reduce the neutron levels to a safe level in the surface laser room. 
Table 1. Magnetic chicane parameters for the BDS Compton polarimeters. The magnet labels given in parenthesis refer to figures 6 and 7 .

\begin{tabular}{|lrlrl|}
\hline Chicane Parameters & Upstream & Polarimeter & Downstream & Polarimeter \\
\hline Chicane Length [m] & 74.6 & & 72.0 \\
Number of magnets & 12 & 6 & 6.0 \\
Magnet Length [m] & 2.4 & 0.4170 & $(1 \mathrm{P}, 2 \mathrm{P})$ \\
& & & 0.6254 & $(3 \mathrm{P}, 4 \mathrm{P})$ \\
Magnetic Field [T] & 0.0982 & $(1 \mathrm{P}-12 \mathrm{P})$ & 0.4170 & $(1 \mathrm{G}, 2 \mathrm{G})$ \\
& & & 11.7 & $(1 \mathrm{P}-3 \mathrm{P})$ \\
& & & 13.2 & $(4 \mathrm{P})$ \\
& 1.25 & $(1 \mathrm{P}-12 \mathrm{P})$ & 14.7 & $(1 \mathrm{G}, 2 \mathrm{G})$ \\
\hline & & & 40.0 & $(1 \mathrm{P}-3 \mathrm{P})$ \\
& 10.0 & $(1 \mathrm{P}-3 \mathrm{P})$ & 54.0 & $(4 \mathrm{P})$ \\
& 20.0 & $(4 \mathrm{P}-9 \mathrm{P})$ & 40.0 & $(1 \mathrm{G}, 2 \mathrm{G})$ \\
\hline & 30.0 & $(10 \mathrm{P}-12 \mathrm{P})$ & & \\
Magnet pole-face width [cm] 1/2-gap [cm] & & & & 20 \\
\hline Dispersion at mid-chicane & & 20 & & \\
for 250 GeV [mm] & & & & \\
\end{tabular}

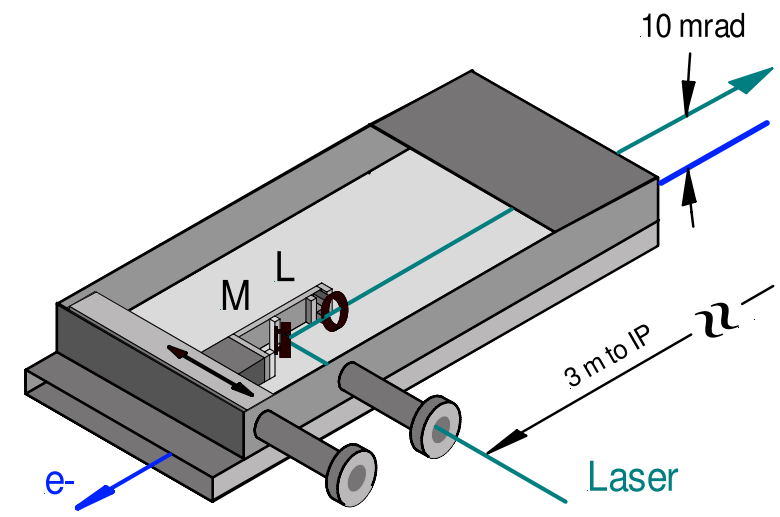

Figure 4. Movable mirror and lens focussing the laser onto the electron beam.

The polarimeters employ magnetic chicanes with the parameters shown in table 1 . The parameters where chosen such that a safe distance from the main beam can be ensured for the Cherenkov detectors while simultaneously avoiding emittance blow-up and keeping the cost at minimum. Both chicanes have been designed to spread the Compton spectrum horizontally over about $20 \mathrm{~cm}$, while allowing to keep a relatively low dispersion of $20 \mathrm{~mm}$ at the mid-chicane point even for a beam energy of $E_{b}=250 \mathrm{GeV}$. The spread of the Compton spectrum does not change and is independent of the beam energy if the magnetic field is kept constant. This leads to a stable position distribution of fixed shape and location at the surface of the Cherenkov detector. However, the Compton IP moves laterally with the beam energy. Figure 4 shows a setup to adjust the laser accordingly, resulting in a maximal dispersion of about $12 \mathrm{~cm}$ at $E_{b}=45.6 \mathrm{GeV}$ and a minimal dispersion of about $1 \mathrm{~cm}$ at $E_{b}=500 \mathrm{GeV}$. 

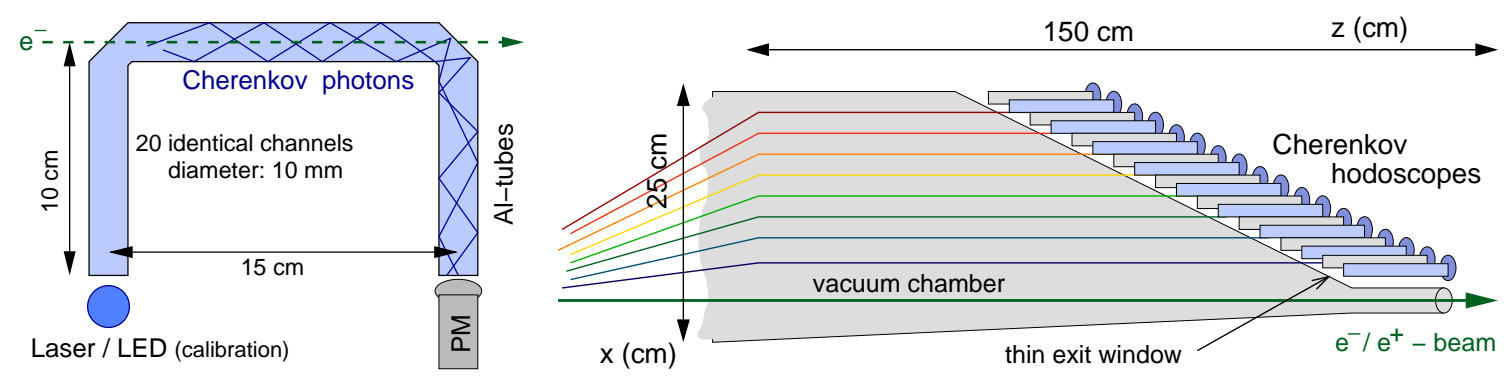

Figure 5. Schematic of a single gas tube (left) and the complete hodoscope array covering the tapered exit window (right) as foreseen for the Cherekov detectors of both polarimeters.

In order to relate the measurements of the polarimeters to the polarization measured at the actual $e^{+} e^{-}$IP, the depolarization along the accelerator and during the collisions has to be modelled in theory and simulations, which have to be confronted finally with real data from the polarimeters and $e^{+} e^{-}$collisions. Current studies predict a depolarization of about $0.2 \%$ for nominal parameters of the ILC beams, i.e. $P\left(e^{-}\right)=80 \%, P\left(e^{+}\right)=30 \%$, dominated by spin precession effects [6].

\subsection{Polarimeter detectors}

Since both BDS polarimters will use Cherenkov detectors, but differ in the requirements, several design options for the detectors are being studied. One uses gas tubes for the radiator with the Cherenkov light detected by conventional photomultipliers (PMs), or newer types such as multianode photomultipliers (MAPMs), both of which are suitable for the upstream and the downstream polarimeter. Another option utilises silicon-based photomultipliers (SiPMs) coupled to quartz fibers as radiator.

Figure 5 illustrates the first design option with one gas-filled detector channel shown on the left-hand side and an arrangement of $\mathscr{O}(20)$ channels covering the entire exit window for the Compton scattered electrons on the right-hand side. The gas tubes have a square cross section of about $1 \mathrm{~cm}^{2}$, where the actual value has to be optimised with respect to the effective area of the finally chosen photodetector. The number of channels has been chosen taking into account the width of the Compton spectrum provided by the chicane, typical photodetector sizes, the resolution required to resolve features like the zero-crossing of the asymmetry and, of course, the cost. One of the Cherenkov gases being considered is perfluorobutane $\left(\mathrm{C}_{4} \mathrm{~F}_{10}\right)$ since it has a high Cherenkov threshold of $10 \mathrm{MeV}$ and does not scintillate from lower energy particles. Other gases with similar properties are also considered. Propane, for example, was chosen for the SLD polarimeter detector [7], but it had the drawback of being flammable.

While the segmented anodes of MAPMs allow independent readout and thus a position resolution even within detector channels, higher cross talk between those multiple anode pads might be an issue and will need to be studied.

Silicon-based PMs have excellent single photon detection capabilities and outmatch conventional PMs in terms of robustness, size and cost. However the quartz fibers constituting the radiator material for this detector option have a much lower Cherenkov threshold of only about $200 \mathrm{keV}$ making them more susceptible to background radiation [8]. This might still be acceptable for the upstream polarimeter, but is less likely to work for the downstream polarimeter due to the much higher backgrounds. 


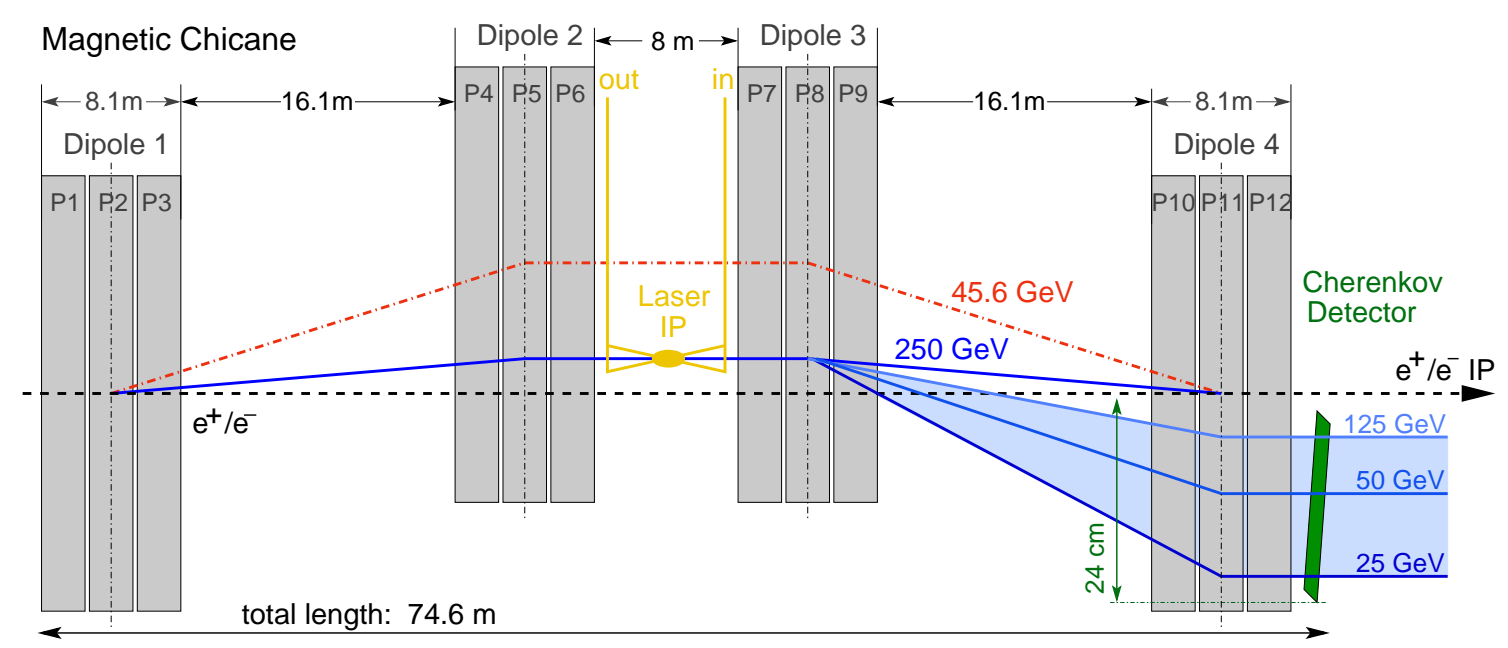

Figure 6. Schematic of the upstream polarimeter chicane.

Linearity and longterm stability of various photodetectors (conventional, MAPMs and SiPMs) are currently studied in an LED test setup, as well as during different testbeam periods with a newly constructed two channel Cherenkov detector prototype [8].

\subsection{Upstream polarimeter}

The upstream Compton polarimeter is located at the beginning of the BDS, upstream of the tuneup dump $1800 \mathrm{~m}$ before the $e^{+} e^{-}$IP. In this position it benefits from clean beam conditions and very low backgrounds. The upstream polarimeter configuration in the RDR is shown in figure 6 . It will provide fast and precise measurements of the polarization before collisions. The beam direction at the Compton IP in both the vertical and horizontal must be the same as that at the IP within a tolerance of $\sim 50 \mu \mathrm{rad}$.

The parameters for the upstream chicane and Cherenkov detector were chosen such that the entire Cherenkov spectrum can be measured for all beam energies while still keeping the Cherenkov detector at a clearance of $2 \mathrm{~cm}$ with respect to the beam pipe.

The upstream polarimeter can be equipped with a laser similar to one used at the TTF/Flash source in operation at DESY. It can have the same pulse structure as the electron beam allowing measurements of every bunch. This permits fast recognition of polarization variations within each bunch train as well as time-dependent effects that vary train-by-train. The statistical precision of the polarization measurement is estimated to be $3 \%$ for any two bunches with opposite helicity, leading to an average precision of $1 \%$ for each bunch position in the train after the passage of only 20 trains ( 4 seconds). The average over two entire trains with opposite helicity will have a statistical error of $\Delta P / P=0.1 \%$.

The RDR design for the upstream polarimeter chicane included capability for a laserwire detector for beam emittance measurements and a machine-protection system (MPS) energy collimator. The combined functionality for these devices in the polarimeter chicane compromised some aspects of the polarimeter capabilities and operation $[4,9]$. Therefore it is now planned to have a dedicated chicane for the upstream polarimeter as shown in figure 6. 
Energy Chicane

$$
\begin{array}{ccc}
\multicolumn{3}{c}{3 \mathrm{E}} \\
1 \mathrm{E} & \mathrm{z} 55.2 \mathrm{~m} & \\
\mathrm{z} \sim 46.8 \mathrm{~m} & \mathrm{zE} \\
& \mathrm{z} 68.8 \mathrm{~m}
\end{array}
$$

Horizontal Bend Magnets

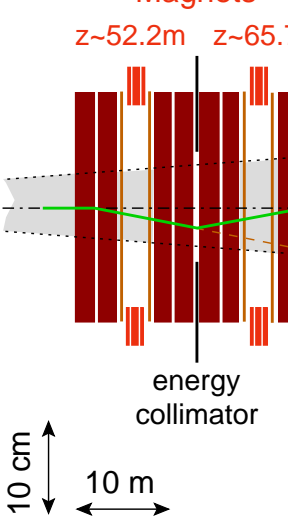

\section{Polarimeter Chicane}

$\begin{array}{cccc}1 \mathrm{P} & 2 \mathrm{P} & 3 \mathrm{P} & 4 \mathrm{P} \\ \mathrm{z} 120.7 \mathrm{~m} & +\mathrm{dz}=20 \mathrm{~m} & +\mathrm{dz}=12 \mathrm{~m} & +\mathrm{dz}=20 \mathrm{~m}\end{array}$

Cherenkov Det. SR-shielding for
Cherenkov det.

Figure 7. Schematic of the ILC extraction line diagnostics for the energy spectrometer and the Compton polarimeter.

\subsection{Downstream polarimeter}

The downstream polarimeter, shown in figure 7 , is located $150 \mathrm{~m}$ downstream of the IP in the extraction line and on axis with the IP and IR magnets. It can measure the beam polarization both with and without collisions, thereby testing the calculated depolarization due to collisions. An example as to how this could be accomplished is given in section 2.4 on page 9 .

A complete conceptual layout for the downstream polarimeter exists, including magnets, laser system and detector configuration [10]. The downstream polarimeter chicane successfully accommodates a detector for the downstream energy spectrometer and provides magnetic elements for the GAMCAL system [10]. In order for the downstream Cherenkov detector to avoid the synchrotron radiation fan from the $e^{+} e^{-}$IP (extending about $15 \mathrm{~cm}$ from the beam pipe, see figure 7), the downstream dipole magnets are larger and have much higher fields. In addition, magnets $3 \mathrm{P}$ and $4 \mathrm{P}$ are operated at higher fields (compared to magnets $1 \mathrm{P}$ and $2 \mathrm{P}$ ) in order to bend the scattered electrons further from the main beam axis. Therefore, two additional magnets $(1 \mathrm{G}$ and $2 \mathrm{G})$ are needed to bring the main beam back to its original trajectory.

The laser for the downstream polarimeter requires high pulse energies to overcome the much larger backgrounds in the extraction line. Three 5-Hz laser systems will be used to generate Compton collisions for three out of 2800 bunches in a train. Each laser is an all solid-state diode-pumped Nd:YAG, with a fundamental wavelength of $1064 \mathrm{~nm}$ that will be frequency-doubled to $532 \mathrm{~nm}$. Each laser will sample one particular bunch in a train for a time interval of a few seconds to a minute, then select a new bunch for the next time interval, and so on in a pre-determined pattern. The Compton statistics are high with more than 1000 Compton-scattered electrons per bunch in a 


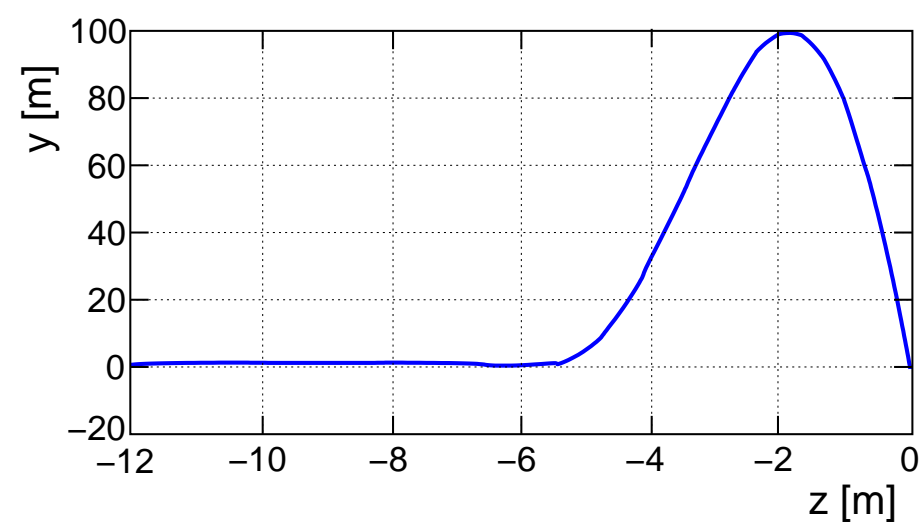

Figure 8. Vertical trajectory of the beam in SiD with anti-DID and $14 \mathrm{mrad}$ crossing angle. The collider IP is located at $z=0 \mathrm{~m}$. (Taken from figure 9 in Reference [11].)

detector channel at the Compton edge. With this design, a statistical uncertainty of less than $1 \%$ per minute can be achieved for each of the measured bunches. This is dominated by fluctuations in Compton luminosity due to beam jitter and laser targeting jitter and to possible background fluctuations. Even though the sampling rate of the downstream polarimeter is much lower than that of the upstream polarimeter, they still give complementary information. The downstream polarimeter can measure the polarization both with and without collisions and is thus able to assess the calculated depolarization due to collisions. On the other hand, the upstream polarimeter can resolve intra-train polarization variations and time-dependent effects varying on a train-by-train basis.

Background studies have been carried out for disrupted beam losses and for the influence of synchrotron radiation (SR). There are no significant beam losses for the nominal ILC parameter set and beam losses look acceptable even for the low power option. An SR collimator protects the Compton detector and no significant SR backgrounds are expected.

\subsection{Impact of crossing angle and IR magnets on polarimetry}

The current ILC design forsees a crossing angle of 14 mrad between the two colliding beams, which means that the beam trajectory and the detector solenoid axis will be misaligned. This causes a vertical deflection of the beam and also impacts the trajectory of low energy pairs produced in the collision [11]. A detector-integrated dipole (DID) can be included in the solenoid to compensate either for the beam trajectory at the IP or the trajectory of low energy pairs as they leave the IR. To reduce backscattering of this pair background into the vertex and tracking detectors at the $e^{+} e^{-}$IP it is preferable to align the trajectory of low energy pairs with the extraction beamline (anti-DID solution). However, this results in a significant vertical beam angle at the IP. An example of this is shown for the SiD detector concept in figure 8.

With the anti-DID solution, additional orbit compensation is needed to achieve the goal of less than $50 \mu \mathrm{rad}$ misalignments between the beam trajectory at the collider IP and the polarimeter Compton IPs. This compensation is energy-dependent and is not easily done by compensating the orbit at the upstream polarimeter with correctors due to tolerances on emittance growth. Corrector compensation is more easily done for the downstream polarimeter. For the upstream polarimeter, it is highly desirable to implement local orbit compensation near the IR to align the incoming vertical 
beam trajectory with the trajectory at the collider IP. Such a scheme looks feasible, but has not yet been fully described [11]. For the downstream polarimeter, the following procedure can be used to set the extraction line corrector magnets:

- Obtain an extraction line reference orbit with the solenoid, anti-DID and correctors off;

- Then use correctors to reproduce the reference orbit as the solenoid and anti-DID are ramped to nominal settings (can compare calculated and actual corrector settings);

- Then adjust correctors to match beam angle at the Compton IP with the collider IP angle (if non-zero).

While this procedure seems suitable, its final precision has yet to be studied in simulations.

\section{Beam energy measurements}

The ILC RDR design provides redundant beam-based measurements of the incoming beam energy, capable of achieving $10^{-4}$ accuracy. The measurements would be available in real time as a diagnostic tool to machine operators and would provide the basis for the determination of the luminosity-weighted center-of-mass energy for physics analyses. Physics reference channels, such as a final state muon pair resonant with the known $Z^{0}$ mass, are then foreseen to provide valuable cross checks of the collision scale, but only long after the data has been recorded.

The two primary methods planned for making precise beam energy measurements are a noninvasive spectrometer based on beam position monitors (BPMs), located upstream of the interaction point just after the energy collimators (figure 1), and a synchrotron imaging detector which is located downstream of the IP in the extraction line to the beam dump (figures 1 and 7). The BPMbased device is modeled after the spectrometer built for LEP II [12], which was used to calibrate the energy scale for the $W$-boson mass measurement, although the parameters of the ILC version are much more tightly constrained by allowances on emittance dilution in the beam delivery system. The synchrotron imaging detector is similar in design to the spectrometer used at SLAC for the SLC program. Both are designed to provide an absolute measurement of the beam energy scale to a relative accuracy of $10^{-4}$ (100 parts per million, ppm). The downstream spectrometer, which observes the disrupted beam after collisions, can also measure the energy spectrum of the disrupted beam.

\subsection{Upstream energy spectrometer}

The RDR includes a BPM-based energy spectrometer, located about $700 \mathrm{~m}$ upstream of the interaction point, just after the energy collimation system. The spectrometer consists of four dipoles which introduce a fixed dispersion of $\eta=5 \mathrm{~mm}$ at the centre. Before, after and at the centre the beam line is instrumented with 2 or more cavity BPMs mounted on translation systems (so that the cavities can always be operated at their electromagnetic centre), shown in figure 9. With the four magnet chicane system systematics associated to the magnets can be investigated, such as hysteresis and residual fields. The four magnet chicane also allows the spectrometer to be operated at different field strengths without disturbing the rest of the machine. It is important that the energy spectrometer be able to make precision energy measurements between $45.6 \mathrm{GeV}$ ( $Z$-pole) and the highest ILC 


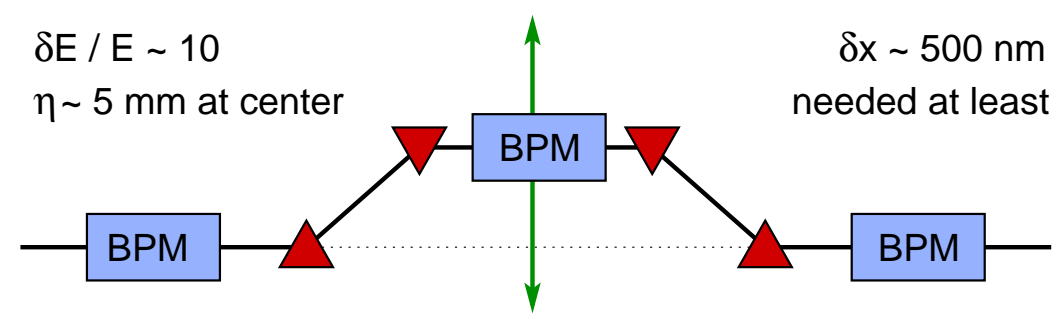

Figure 9. Schematic for the upstream energy spectrometer using BPMs.

energy of $500 \mathrm{GeV}$. When operating the spectrometer with a fixed dispersion over the whole energy range, a BPM resolution of $0.5 \mu \mathrm{m}$ is required. Cavity beam position monitors can achieve the required single shot accuracy, even significantly better accuracy of $20 \mathrm{~nm}$ has been achieved [13]. However, for a fixed dispersion, the spectrometer magnets will need to operate at low magnetic fields when running at $45.6 \mathrm{GeV}$ where the magnetic field measurement may not be accurate enough. On the other hand, a BPM resolution of $20 \mathrm{~nm}$ would allow the chicane dipoles to be run at the same magnetic field for both the Z-pole and highest energy operation. The parameters of the upstream energy spectrometer have been choosen taking into account the required precision of the energy measurement, the precisions achievable for the magnetic field measurements and the BPM measurements, emittance preservation, and the shortest possible chicane length (for cost reasons).

The absolute energy measurement requires that the beam orbit with no field is measured. There is a research program to determine how to perform accurate magnetic field measurements for low fields, or even zero field as needed for the absolute beam energy measurements.

A prototype test setup for such an instrument was commissioned in 2006 and 2007 in the T474 experiment in the End Station A beamline at SLAC. The setup involved four dipole magnets and high-precision RF cavity BPMs in front, behind and in between the magnets. ESA test beams operated at $10 \mathrm{~Hz}$ with a bunch charge of $1.6 \cdot 10^{10}$ electrons, a bunch length of $500 \mu \mathrm{m}$ and an energy spread of $0.15 \%$, i.e. with properties similar to ILC expectations. The beam energy is directly deduced from the beam offset measurements normalized to the $5 \mathrm{~mm}$ dispersion (same dispersion as for the present ILC baseline energy spectrometer). When combining all the BPM stations to measure the precision of the orbit over the whole ESA-chicane beamline, a resolution of $0.8 \mu \mathrm{m}$ in $x$ and $1.2 \mu \mathrm{m}$ in $y$ was achieved. The system turned out to be stable at the micron level over the course of one hour, which would translate to an energy precision of $200 \mathrm{ppm}$ [14]. Although the single pulse resolution is sufficient for the ILC physics goals, systematic errors associated with energy loss due to the downstream final focus system to the interaction has yet to be evaluated. Additional studies are being conducted to measure and correct for motions much smaller than 1 micron and over time periods greater than one hour [15].

\subsection{Extraction line energy spectrometer}

At the SLC, the WISRD (Wire Imaging Synchrotron Radiation Detector) [16] was used to measure the distance between two synchrotron stripes created by vertical bend magnets which surrounded a precisely-measured dipole that provided a horizontal bend proportional to the beam energy. This device achieved a precision of $\Delta E_{b} / E_{b} \sim 2 \cdot 10^{-4}(200 \mathrm{ppm})$, where the limiting systematic errors 
were due to relative component alignment and magnetic field mapping. The ILC Extraction-Line Spectrometer (XLS) design [17] is largely motivated by the WISRD experience. The energy spectrometer will make precision energy measurements between $45.6 \mathrm{GeV}$ (Z-pole) and the highest ILC energy of $500 \mathrm{GeV}$. The RDR extraction line design parameters are the result of a delicate optimization between the downstream spectrometer and polarimeter needs, plus the primary requirement of the extraction line to safely transport the highly disrupted outgoing beam to the dump. The interleaved design of the polarimeter and spectrometer elements, as well as the dipole bending strengths and required apertures were also constrained by overall considerations of cost and feasibility.

The analyzing dipole for the XLS is provided by a vertical chicane just after the capture quad section of the extraction line, about $55 \mathrm{~m}$ downstream of the interaction point (see figure 7). The chicane provides a $\pm 2 \mathrm{mrad}$ vertical bend to the beam and in both legs of the chicane horizontal wiggler magnets are used to produce the synchrotron light needed to measure the beam trajectory. The optics in the extraction line is designed to produce a secondary focus about $150 \mathrm{~m}$ downstream of the IP, which coincides with the center of the polarimeter chicane and the Compton interaction point. The synchrotron light produced by the wigglers will also come to a vertical focus at this point, and position-sensitive detectors in this plane arrayed outside the beampipe will measure the vertical separation between the synchrotron stripes.

With a total bend angle of $4 \mathrm{mrad}$, and a flight distance of nearly $100 \mathrm{~m}$, the synchrotron stripes will have a vertical separation of $400 \mathrm{~mm}$, which must be measured to a precision of $40 \mu \mathrm{m}$ to achieve the target accuracy of $10^{-4}$. In addition to the transverse separation of the synchrotron stripes, the integrated bending field of the analyzing dipole also needs to be measured and monitored to a comparable precision of $10^{-4}$. The distance from the analyzing chicane to the detectors needs to only be known to a modest accuracy of $1 \mathrm{~cm}$. For the XLS spectrometer, it has been proposed to use an array of radiation-hard $100 \mu \mathrm{m}$ quartz fibers. These fibers do not detect the synchrotron light directly, but rather detect Cherenkov radiation from secondary electrons produced when the hard photons interact with material near the detector. At ILC beam energies, the critical energy for the synchrotron radiation produced in the XLS wigglers is several tens of $\mathrm{MeV}$, well above the pair-production threshold, and copious numbers of relativistic electrons can be produced with a thin radiator in front of the fiber array. The leading candidates for reading out these fibers are multi-anode PMs from Hamamatsu, similar in design to those used in scintillating fiber calorimeters. The advantage of this scheme over wires (as used in the SLC energy spectrometer) is to produce a reliable, passive, radiation-hard detector which does not suffer from cross talk or RF pickup, and still allows for easy gain adjustment and a large dynamic range.

The energy spectrum of the beam after collision contains a long tail as a result of the beambeam disruption in the collision process. This disrupted beam spectrum is not a direct measure of the collision energy spectrum, but it is produced by the same physical process, and direct observation of this disrupted tail will serve as a useful diagnostic for the collision process. The position-sensitive detector in the XLS is designed to measure this beam energy spectrum down to $50 \%$ of the nominal beam energy. Near the peak, for a beam energy of $E_{b}=250 \mathrm{GeV}$, each 100micron fiber spans an energy interval of $125 \mathrm{MeV}$. Given a typical beam energy width of $0.15 \%$, this means the natural width of the beam energy will be distributed across at least a handful of fibers, which will allow the centroid to be determined with a precision better than the fiber pitch, and some information about the beam energy width can be extracted as well. 


\subsection{Alternative methods for energy measurements}

$R \& D$ on three alternative methods for precise beam energy measurements with $100 \mathrm{ppm}$ accuracy is being carried out by different groups. The first method utilizes Compton backscattering, a magnetic spectrometer and precise position measurements of the electron beam, the centroid of the Compton photons and the kinematic edge of the Compton-scattered electrons [18, 19]. The spectrometer length needed is about $30 \mathrm{~m}$ and would be located near the upstream polarimeter (or may utilize the upstream polarimeter chicane). Precise position measurements approximately $25 \mathrm{~m}$ downstream of an analysis magnet are needed with accuracies of $1 \mu \mathrm{m}$ for the Compton photons, $10 \mu \mathrm{m}$ for the Compton edge electrons and $0.5 \mu \mathrm{m}$ for the beam electrons.

The second method utilizes the SR emitted in the dipole magnets of the upstream BPM-based spectrometer [20]. Accurate determination of the edges of the SR fan is needed. Studies include a direct measurement of the SR fan as well as the use of mirrors to deflect soft SR light to detectors located away from the beamline. Novel high spatial resolution detectors are considered.

A third method relies on the Resonance Absorption method [21, 22]. Under certain conditions, laser light can be absorbed by beam particles when both co-propagate in close proximity in a solenoid. The beam energy can be infered from the measured dependence of light absorption on the magnetic field and laser wavelength.

\section{Summary}

Concepts for high precision polarization and energy measurements at the ILC exist. These concepts have resulted in detailed system layouts that are included in the current description for the Beam Delivery System as specified in the Reference Design Report. The RDR includes both upstream and downstream polarimeters and energy spectrometers for both beams. This provides needed complementarity and redundancy for achieving the precision required, with adequate control and demonstration of systematic errors.

Different beam conditions have led to different layouts for the upstream diagnostic systems compared to the respective downstream ones. However, each system is designed to provide precise measurements at a large range of beam energies from $45.6 \mathrm{GeV}$ at the $Z^{0}$ pole up to $250 \mathrm{GeV}$ or even up to $500 \mathrm{GeV}$ as discussed for an upgrade option. A workshop was held in 2008 on ILC polarization and beam energy measurements, which resulted in a set of recommendations for the ILC design and operation. Most of them will be implemented in the next revision of the ILC baseline design.

Work is continuing during the ILC engineering design phase to further optimize the polarimeter and energy spectrometer concepts and fully implement them in the ILC. This includes consideration for alternative methods, detailed design and cost estimates, and prototype and test beam activities.

\section{Acknowledgments}

Part of this work has been supported by the U.S. Department of Energy contract number DE-AC0276SF00515 and by the Deutsche Forschungsgemeinschaft via grant Li-1560/1-1. 


\section{References}

[1] G.A. Moortgat-Pick et al., The role of polarized positrons and electrons in revealing fundamental interactions at the linear collider, Phys. Rept. 460 (2008) 131 [hep-ph/0507011].

[2] N. Phinney, N. Toge and N. Walker (eds.), International Linear Collider Reference Design Report. III. Accelerator, available at http://www.linearcollider.org/cms/?pid=1000437 (2007).

[3] K.C. Moffeit et al., Spin Rotation Schemes at the ILC for Two Interaction Regions and Positron Polarization with both Helicities, LCC-0159, SLAC-TN-2005-045 (2005);

K.C. Moffeit, D. Walz and M. Woods, Spin Rotation at lower energy than the damping ring, ILC-NOTE-2008-040, IPBI TN-2008-1 (2008);

K.C. Moffeit, Spin Rotation before the Damping Ring, in Proceedings of Workshop on Polarization and Beam Energy Measurements at ILC, Zeuthen Germany, April 9-11 2008, https://indico.desy.de/conferenceDisplay.py?confId=585 [IPBI TN-2008-3].

[4] B. Aurand et al., Executive Summary of the Workshop on Polarization and Beam Energy Measurements at the ILC, Workshop on Polarization and Beam Energy Measurements at ILC, Zeuthen Germany, April 9-11 2008, https://indico.desy.de/conferenceDisplay.py?confId=585 [arXiv:0808.1638].

[5] M.L. Swartz, A complete order- $\alpha^{3}$ calculation of the cross section for polarized Compton scattering, Phys. Rev. D 58 (1998) 014010 [hep-ph/9711447].

[6] I.R. Bailey et al., Depolarization and Beam-Beam Effects at the Linear Collider, EUROTeV-Report-2008-026 (2008).

[7] SLD collaboration, K. Abe et al., First measurement of the left-right cross-section asymmetry in Z boson production by $e^{+} e^{-}$collisions, Phys. Rev. Lett. 70 (1993) 2515; An improved direct measurement of leptonic coupling asymmetries with polarized $Z$ bosons,

Phys. Rev. Lett. 86 (2001) 1162 [hep-ex/0010015]; An improved measurement of the left-right $Z^{0}$ cross- section asymmetry, Phys. Rev. Lett. 78 (1997) 2075 [hep-ex/9611011];

R. Elia, Measurement of the Left-Right Asymmetry in Z Boson Production by Electron-Positron Collisions, SLAC-Report-429 (1994);

R. King, A Precise Measurement of the Left-Right Asymmetry of Z Boson Production at the SLAC Linear Collider, SLAC-Report-452, 1994;

A. Lath, A Precise Measurement of the Left-Right Cross Section Asymmetry in Z Boson Production, SLAC-Report-454 (1994);

E. Torrence, Search for Anomalous Couplings in the Decay of Polarized Z Bosons to Tau Lepton Pairs, SLAC-Report-509 (1997).

[8] C. Bartels, C. Helebrant, D. Kafer and J. List, Compton Cherenkov Detector Development for ILC Polarimetry, in Proceedings of LCWSO8, Chicago Illinois, U.S.A., November 16-20 2008, arXiv:0902.3221.

[9] J. List and D. Kafer, Improvements to the ILC Upstream Polarimeter, in Proceedings of LCWSO8, Chicago Illinois, U.S.A., November 16-20 2008, arXiv: 0902.1516;

J. List, The ILC Upstream Polarimeter, presentation at the Workshop on Polarization and Beam Energy Measurements at ILC, Zeuthen Germany, April 9-11 2008, https://indico.desy.de/conferenceDisplay.py?confId=585.

[10] K.C. Moffeit et al., Proposal to modify the polarimeter chicane in the ILC 14 mrad extraction line, SLAC-PUB-12425, IPBI TN-2007-1 (2007);

K.C. Moffeit, Downstream Extraction Line Polarimeter, in Proceedings of Workshop on Polarization and Beam Energy Measurements at ILC, Zeuthen Germany, April 9-11 2008, https://indico.desy.de/conferenceDisplay.py?confId=585 [IPBI TN-2008-5]. 
[11] A. Seryi, T. Maruyama and B. Parker, IR Optimization, DID and anti-DID, SLAC-PUB-11662 (2006).

[12] LeP Energy Working Group collaboration, R. Assmann et al., Calibration of centre-of-mass energies at LEP2 for a precise measurement of the W boson mass, Eur. Phys. J. C 39 (2005) 253 [hep-ex/0410026].

[13] S. Walston et al., Performance of a High Resolution Cavity Beam Position Monitor System, Nucl. Instrum. Meth. A 578 (2007) 1.

[14] M. Slater et al., Cavity BPM system tests for the ILC energy spectrometer, Nucl. Instrum. Meth. A 592 (2008) 201.

[15] ATF2 GRouP collaboration, B.I. Grishanov et al., ATF2 proposal, available from http://lcdev.kek.jp/ATF2/proposal/.

[16] F. Rouse et al., Measuring the Mass and Width of the $Z^{0}$ : The Status of the Energy Spectrometers, SLAC-PUB-4977 (1989).

[17] E. Torrence, Downstream Synchrotron Radiation Stripe Spectrometer Status, presentation at the Workshop on Polarization and Beam Energy Measurements at ILC, Zeuthen Germany, April 9-11 2008, https://indico.desy.de/conferenceDisplay.py?confId=585.

[18] N. Muchnoi, H.J. Schreiber and M. Viti, ILC Beam Energy Measurement by means of Laser Compton Backscattering, Nucl. Instrum. Meth. A 607 (2009) 340 [arXiv : 0812.0925].

[19] N. Muchnoi, Proposal for Eb Measurement at Novosibirsk Using Compton Backscattering, presentation at the Workshop on Polarization and Beam Energy Measurements at ILC, Zeuthen Germany, April 9-11 2008, https://indico.desy.de/conferenceDisplay.py?confId=585.

[20] K. Hiller, H.J. Schreiber, R. Makarov, E. Syresin and B. Zalikhanov, ILC beam energy measurement based on synchrotron radiation from a magnetic spectrometer, Nucl. Instrum. Meth. A 580 (2007) 1191.

[21] R. Melikian, Development of the Theory of Measurement of Electron Beam Absolute Energy by Resonance Absorption Method, presentation at the Workshop on Polarization and Beam Energy Measurements at ILC, Zeuthen Germany, April 9-11 2008, https://indico.desy.de/conferenceDisplay.py?confId=585.

[22] A. Ghalumyan, Experiment Proposal for Eb Measurement using the Resonance Absorption Method, presentation at the Workshop on Polarization and Beam Energy Measurements at ILC, Zeuthen Germany, April 9-11 2008, https://indico.desy.de/conferenceDisplay.py?confId=585. 